\title{
Economic Analysis of Energy Storage Business Models
}

\author{
Gavin Fong \\ Dept. of Electrical and Electronic \\ Engineering \\ Imperial College London
}

\author{
Roberto Moreira \\ Dept. of Electrical and Electronic \\ Engineering \\ Imperial College London \\ roberto.m@imperial.ac.uk
}

\author{
Prof. Goran Strbac \\ Dept. of Electrical and Electronic \\ Engineering \\ Imperial College London
}

\begin{abstract}
The increasing penetration of renewable energy systems and the electrification of heat and transport sectors in the UK have created business opportunities for flexible technologies, such as battery energy storage (BES). However, BES investments are still not well understood due to a wide range and debatable technology costs that may undermine its business case. In this context, an economic analysis will be established to assess the economic viability of current BES business models, particularly associated with multiple service portfolios. Our model quantifies the net present values (NPVs) and payback periods of BES investments considering various business models and state-of-theart BES technologies. We determine the commercial viability associated with different BES technologies and business models. The developed model compares different technology costs, business models (i.e. portfolio of services provided) and BES lifetimes to perform a comprehensive economic analysis on the business case for investing in BES. Several case studies under current GB market arrangements demonstrate that BES investment associated with multi-service business models offers the best financial benefits to storage investors and achieve payback periods within 10 years' lifetime.
\end{abstract}

Index Terms - Energy storage, Multi-service business models, Net present value, Payback period, Power System Economics.

\section{INTRODUCTION}

United Kingdom (UK) government's commitments towards achieving $80 \%$ reduction in carbon emissions by 2050 (relative to 1990 levels) [1] and facilitate the transition towards a lowcarbon electricity industry has contributed to an increase in penetration of renewable generation in the UK and in Europe. In this setting, the UK power system faces various challenges; significant increase in low capacity value of intermittent generation as well as electrification of transport and heat sectors which is likely to result in a drastic increment in peak demand, thus reducing the utilisation of less flexible generation infrastructure and electricity network assets. As a consequence, the system integration costs are expected to increase. A costefficient way to cater for this increasing peak demand is to deploy flexible technologies, such as interconnectors, demand response schemes and energy storage technologies that can potentially support system operation and maintain the grid frequency within standard limits [2].
Nevertheless, developing a financially robust storage business case under the current GB market conditions remains a huge challenge for stakeholders and policy makers. Suitable investment framework that adequately remunerates storage owners for the services provided are yet to be introduced [3]. Furthermore, current legislation that fails to adequately classify and define the role of energy storage has subjected storage owners to excessive charges (e.g. double charging associated with renewable levies), thus hindering the investment and deployment of battery energy storage (BES) technologies as reported in [4]. Hence, a BES investment framework that could provide positive return of investment (ROI) to storage investors under current market arrangements is necessary to promote BES deployment. In this paper, we present an economic analysis associated with a multi-service business model framework for BES which offers multiple simultaneous services to the various market sectors of the electricity industry. The model, offers an economic analysis from a stakeholder perspective and assuming a price taker approach, i.e. the model is applied to a single BES asset and disregards any potential impact on market conditions (and services' prices) due to its own operational policies or from other competing technologies.

\section{LITERATURE REVIEW AND CONTRIBUTION}

Energy storage can provide multiple services to various market sectors of the electricity industry, including participation in the energy market and seizing arbitrage opportunities, primary frequency regulation services, defer/postpone network reinforcements as well as enhancing power quality and network reliability, among many others which can facilitate a costeffective transition towards a low carbon electricity industry [2]. The various benefits and applications associated with energy storage systems are widely discussed in [5]-[7] and the different interactions (i.e. synergies and conflicts) between services are discussed in [8]. This demonstrates the role and benefits that energy storage systems can offer to future decarbonised energy markets.

In the particular case of BES, various studies have been conducted to evaluate the economic viability of different applications for these technologies. The application of Li-ion battery to provide primary frequency regulation service in the Danish electricity market was studied in [9] and the economic profitability of the investment was analysed with respect to 
different BES capacities. The study has informed that primary frequency regulation service provided by Li-ion batteries is profitable in the Danish electricity market and can yield a positive net present value (NPV) over a period of 20 years. However, a BES investment analysis over a period of 20 years is potentially too long and will expose storage investors to high risks due to BES degradation effects and market uncertainties.

The economic viability of BES to electricity consumers under a Time-of-Use (ToU) tariff was analyzed in [10] by comparing the total system cost with and without storage operation. The results from [10] have informed that operation of BES with a ToU tariff would not be profitable to electricity consumers considering current cost and market conditions. Hence, in this study, we propose a BES investment model and perform a costsensitivity analysis to determine its economic viability with respect to various BES technology costs under current market arrangements. In another study, the authors of [11] have performed an economic analysis of BES providing single services, namely: load levelling or primary frequency regulation to identify which service can offer the highest financial benefit to storage owners. The NPV and payback period for each service was evaluated and the results have informed that primary frequency regulation is the most profitable service, which generated a net profit of $0.6 \mathrm{M} €$ and a payback period of approximately 11 years for lead-acid technologies. However, lead acid batteries are associated with typical low round-trip efficiencies and short lifetimes which may result in high degradation levels due to its operation for multi-service business models (although its capital cost is relatively low when compared to other technologies), and thus increasing the operation and maintenance (O\&M) costs.

In contrast to single service business models, the authors in [12] have analyzed the potential (simultaneous) provision of various services for a single BES asset; the study coordinates provision of multiple services to various stakeholders and maximises the profit for storage owners. The results from [12] have indicated that the revenue associated with a multi-service business model is approximately 1.5 times higher than that of a single service business model (e.g. provision of frequency response service alone when compared to multiple services), although the study fails to consider investment costs and potential degradation costs for an adequate economic analysis.

To date, storage owners (and stakeholders) are yet to be provided with a suitable and informative economic analysis regarding the viability of BES investments which provide multiple services to the electricity market. In this setting, our study will focus on analysing the economic viability of high performance BES (e.g. Li-ion battery) for various business models, including provision of energy arbitrage, balancing services (e.g. frequency response and reserve) and network services (e.g. peak demand shaving). Therefore, an investment model will be developed to evaluate the NPV and payback period of various BES business models, thus informing potential storage investors regarding current challenges such as market conditions, technology cost and lifetime that may undermine a cost-effective BES investment. The developed model will take into account the current developments in BES business models such as the energy storage centric model proposed in [12] as well as alternative business models with single services, different capital costs and lifetimes.

\section{METHODOLOGY}

A step by step diagram describing the methodology for performing the economic analysis for various BES business models is presented in Fig. 1. Firstly, the necessary data is defined for the BES centric model in [12] using FICO Xpress optimisation software, for example electricity price, availability prices for balancing services, load demand and timing of response and reserve windows. Next, the output from the BES centric model developed in [12] (i.e. total revenue) is used as an input (along with other parameters) for the model presented herein. Technology cost, lifetime, O\&M costs and BES capacity are also required for the analysis, only then the NPV and payback period for a multi-service business model can be determined. Similar steps are repeated for other business models by manipulating the input data to the BES centric model (i.e. selection of services to be provided). Finally, the results are compared to determine the most economically viable business model.

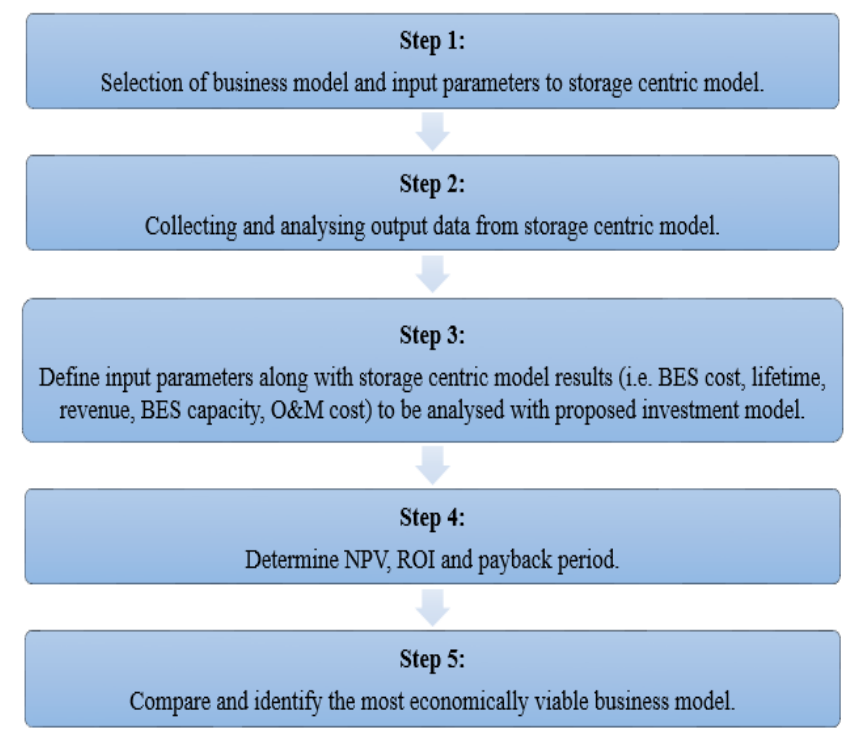

Fig. 1. Research methodology.

Note that in this context, as the analysis is developed with respect to the BES centric model built in [12], therefore most of the input data for revenue calculation are retrieved from [12]. For the purpose of this analysis, we also assumed that the revenue stream generated is constant throughout the entire lifetime of BES and disregards any strategic market behavior capable of changing market prices (i.e. the model assumes a price taker approach).

\section{A. Net Present Value (NPV)}

NPV can be defined as the summation of the present values of cash flows over a pre-defined period of time, according to [13]. In this study, NPV is calculated using (1), throughout the entire assumed BES lifetime, t.

$$
\mathrm{NPV}=\sum_{\mathrm{n}=0}^{\mathrm{t}}\left(\mathrm{F}_{\mathrm{n}} \times \frac{1}{(1+\mathrm{i})^{\mathrm{n}}}\right)
$$


This way, $F_{n}$ denotes future cash flows associated with the revenue obtained from services' provision and i denotes the interest rate considering inflation and nominal discount rate.

\section{B. Payback Period}

The payback period method can be applied together with the NPV method to analyse the economic viability of BES investments [13]. It is used to determine the time required for storage investors to fully recover their invested capitals. This method is only applicable for business models that yield positive NPVs; if the NPV is negative, it is unlikely for storage investors to recover their investments and thus will incur a loss. In this study, the discounted payback period, $\mathrm{P}$ which takes into account the time value of money [14] can be calculated using (2).

$$
\sum_{\mathrm{n}=0}^{\mathrm{t}}\left(\mathrm{F}_{\mathrm{n}} \times \frac{1}{(1+\mathrm{i})^{\mathrm{P}}}\right)=0
$$

\section{RESULTS AND DISCUSSION}

\section{A. Input Data and Modelling Considerations}

For the GB case studies presented herein, the input data used on the storage centric model in [12], is summarized as follows:

- Charge/discharge capacity of BES: $6 \mathrm{MW}$

- Energy capacity: $10 \mathrm{MWh}$

- $\quad$ BES efficiency: $90 \%$

- Technology cost: $300 £ / \mathrm{kWh}$

In addition, the model uses real time series of historic GB market prices (for the energy and balancing market) and real metering demand data from a primary substation in UK. The investment model presented herein, assumes a nominal discount rate and inflation rate of $6 \%$ and $2 \%$ respectively. In addition, the annual operation and maintenance (O\&M) costs were assumed to be $0.5 \%$ CAPEX for the first year and $1 \%$ CAPEX from second year and onwards.

Note that in this analysis, the operational effects on battery cycle-life and calendar life associated with different services and business models were not considered in the model. However, a brief analysis is included in section IV.E to estimate the impacts of battery degradation on different BES services.

\section{B. Revenue of Multi-Service Business Model}

The monthly revenues obtained from different BES services for a multi-service business model (with full services provision) are shown in Fig. 2 per season and account for a total annual revenue of approximately 40,000 £/MWh/annum. Among all, frequency response service has contributed with the highest revenue (irrespective of season), with a revenue which is twice than the combined revenue of the other three services (i.e. energy arbitrage, reserve and network services). It is important to note that provision of frequency response and reserve was limited to time windows pre-defined by the system operator, following GB market framework. In this setting, the revenue associated with frequency response service follows a conservative estimation and can more than double since provision of this service was limited to morning periods. This shows the importance that frequency response services have on the business case of energy storage.

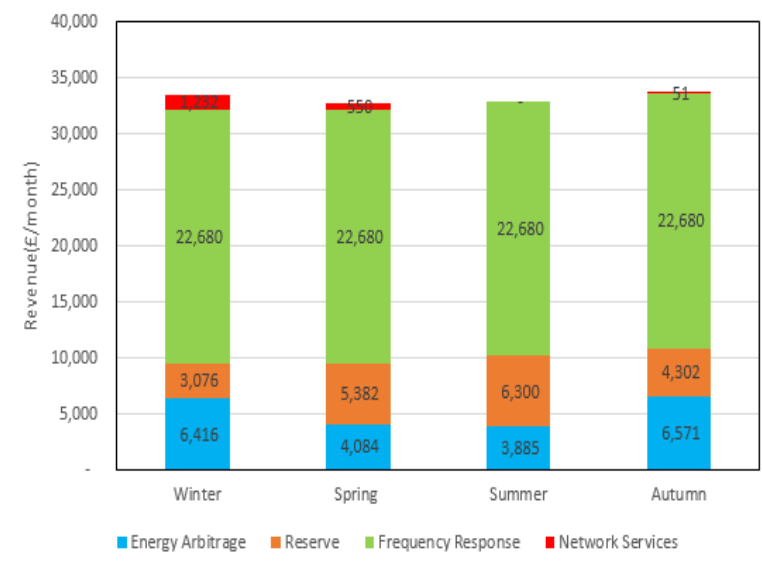

Fig. 2. Monthly revenue of BES services.

Note that no contribution is recorded for network services in summer, and this is because the local (distribution) peak demand is relatively low and therefore network congestion problems rarely occur with summer demand. Nevertheless, in winter and spring seasons the service becomes relevant to ensure security of supply.

A price taker approach was assumed for the analysis and thus the prices for all services are fixed irrespective of BES market penetration or strategic participation. This assumption is justified considering the relative small scale of a BES technology (typically below $10 \mathrm{MW}$ ) comparatively to the total generation capacity in the GB market, i.e. approximately 60 GW according to [1].

\section{Economic Profitability of Various Business Models}

To understand the economic profitability of different combination of services and thus different business models, five cases were evaluated, namely:

- Full Service Provision, comprising provision of energy arbitrage, frequency response, reserve and network services;

- Energy Arbitrage \& Frequency Response, covering provision of energy arbitrage and frequency response service;

- $\quad$ Energy Arbitrage \& Network Services, covering provision of energy arbitrage and network services;

- Frequency Response service only;

- Balancing \& Network Services, comprising provision of reserve, frequency response and network services.

Fig. 3 presents the NPV associated with each business model considered over a period of 20 years. 


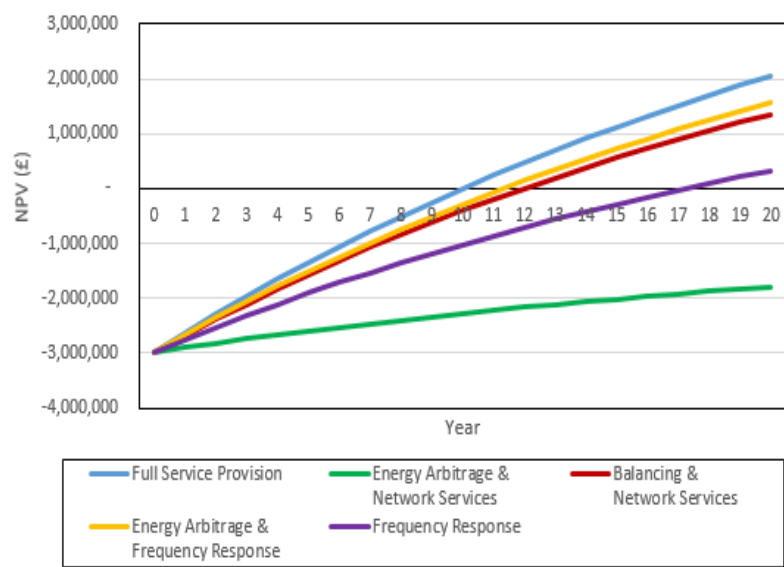

Fig. 3. NPV curves for various business models over a 20-year period.

The results show that considering a multi-service business model with full service provision yields the highest NPV (based on a 20 year BES lifetime) and the invested capital can be recovered within 10 years. This is a more promising result for storage investors as compared to a single service business model (i.e. primary frequency regulation) studied in [9], as our results yield a positive NPV in a shorter period than the latter

Our analysis also demonstrated that a $300 £ / \mathrm{kWh}$ BES is not an economically viable investment for seizing energy arbitrage opportunities under current energy market conditions as its revenue is insufficient to achieve a payback period within the technology lifetime. In fact, for a BES to achieve a positive NPV when seizing arbitrage opportunities would require more than 100 years. A similar result, with a significantly long payback period, is shown for the business model considering energy arbitrage and network services since the low revenue associated with both services is not sufficient to justify the intensive capital expenditure. Note that the substantially higher revenue from provision of frequency response only, leads to a positive NPV in 18 years, which demonstrates the significant role of frequency response service.

The payback period for each business model is summarized in Fig.4.

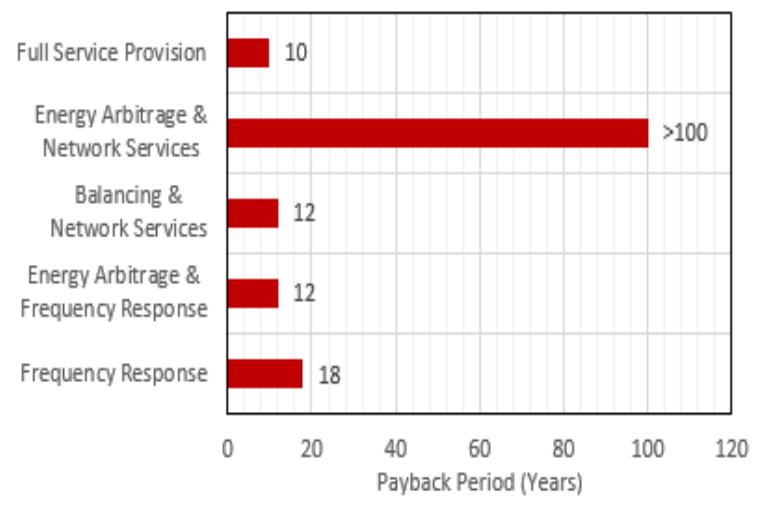

Fig. 4. Payback period for each business model.

\section{Impact of Technology Costs on Payback Period}

In order to achieve an unbiased analysis with respect to technology costs, this section introduces a sensitivity analysis on different technology costs and their impact on the payback period for the full service business model. Fig. 5 shows the different payback periods for different technology costs.

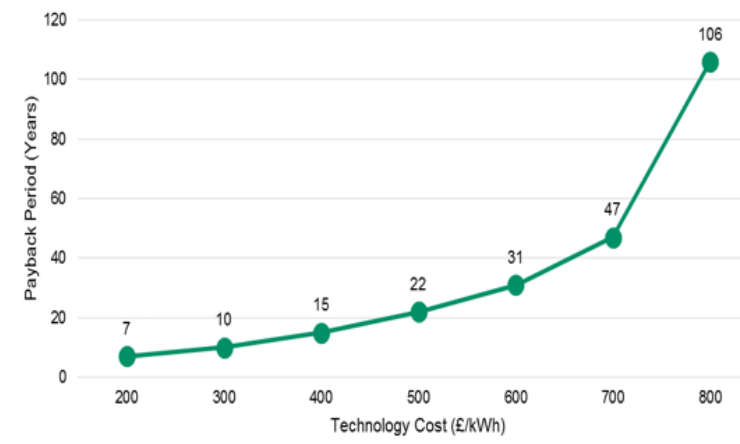

Fig. 5. Payback period for different technology costs.

The results show that for a $400 £ / \mathrm{kWh}$ BES it takes 15 years to recover the invested capital, although this would require adequate management of degradation effects. As the BES cost approaches $500 £ / \mathrm{kWh}$, it would be difficult to recover the investment since the typical lifetime of a Li-ion battery falls between 15 to 20 years, according to [15], whereas the return on investment is achieved in 22 years. This analysis shows that technology cost and lifetime are two major aspects that affect the economic viability of BES investments; a low cost BES may prove economically inefficient if its lifetime is too short, whereas a high cost BES may prove economically efficient if its lifetime is slightly longer.

\section{E. Estimation of BES Cycle-life}

The analysis and results presented in Fig. 3 assume no degradation effects due to different operation policies with each service (and business model). In this section however, we analyse the potential impact that frequent cycling of battery may have on shortening the BES lifetime, and thus compromise an adequate return of investment. Based on Liion technology and typical cycle-life data from a BES manufacturer presented in Fig. 6, the work carried out and presented herein will analyse the impact that different services have on the cycle-life of a BES.

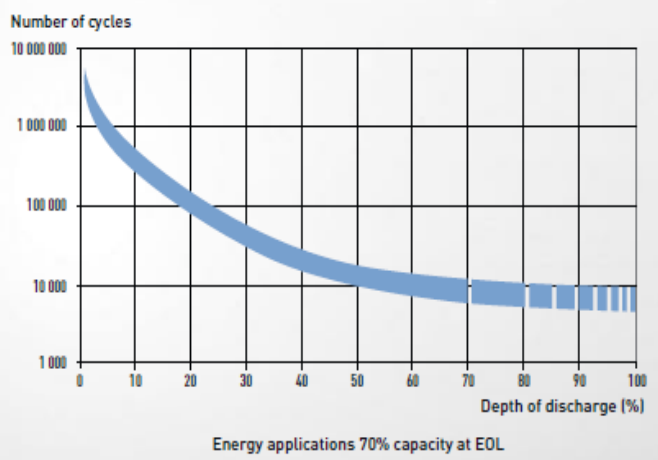

Fig. 6. Typical cycle-life of Li-ion BES [16] 
The cycle-life approximation of Li-ion BES for each business model will consider the depth of discharge (DOD) and number of charge/discharge cycles, similar to method reported in [17]. Initially, the daily average state of charge (SOC) of storage is determined for each business model and thus, the average DOD can be estimated using (3).

$$
\text { Average DOD (\%) }=100 \% \text { - Average SOC }(\%)
$$

This way, we can determine the daily average of a battery DOD considering a specific business model. Then, based on Fig. 6, the battery cycle-life is converted from cycles to years using (4).

$$
\text { BES cycle life (years) }=\frac{\text { Total life cycles }}{\text { Number of cycles per day } \times \text { Number of days per year }}
$$

To consider the potential utilisation in real time of balancing services, i.e. the utilisation of reserve and frequency response due to real time frequency deviation events in the system, two scenarios (best case and worst case) are considered for each business model involving balancing services. Note that although the scheduled operation for the BES considers charge/discharge actions to adequately ensure that energy levels are sufficient to deliver frequency response or reserve services, real time operation and potential oscillations due to positive/negative frequency deviations are not considered and need therefore to be estimated. For instance, if system operator instructs the delivery of reserve service once or twice a day, BES degradation will be significantly different. Fig. 7 shows, for the worst and best case scenarios, an estimated cycle-life for BES considering the different business models.

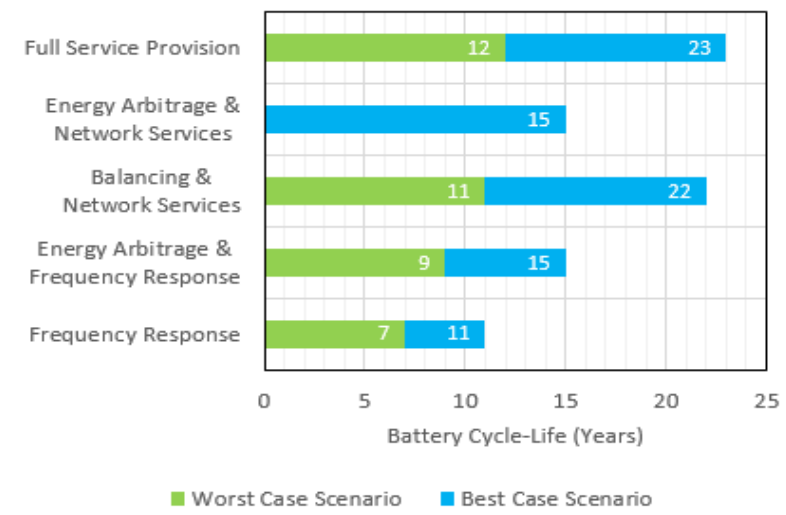

Fig. 7. Estimated Li-ion BES cycle-life.

The results in Fig. 7 show that using a Li-ion BES for the full services business model it not only yields the highest revenue and better business case but also offers the longest lifetime, between 12 to 23 years. This result demonstrates that besides providing the highest revenue, and thus the shortest payback period, a multiple service business model considering energy arbitrage, provision of balancing services and network services also yields the lowest degradation levels on the battery cyclelife. This result is explained by the fact that provision of multiple services allows for BES (power and energy) capacities to be shared and thus materialising the synergies between the multiple services. In our analysis, considering a full service provision would result in less charge/discharge cycles and longer periods of SOC at approximately 50\%, which directly lead to lower degradation levels. In contrast, provision of frequency response service only, would also result in a high revenue albeit increased degradation levels associated with regular charge/discharge cycles.

As seen in Fig. 4, it takes approximately 18 years to recover the CAPEX and OPEX of the BES for a business model which considers provision of frequency response only. Comparatively to the study carried out in [11], which reported a payback period of 11 years for primary frequency regulation involving lead-acid BES, our analysis provides a longer payback period (18 years). Though note that provision of frequency response was limited to the early hours of the morning (as indicated by [12]) and thus following a conservative approach. This leads to significantly lower revenues and potentially to longer payback periods. In addition, different market and regulatory conditions as well as technology costs also play an important role in justifying the difference between the payback periods.

For the case of Balancing \& Network Services and Energy Arbitrage \& Frequency Response business model, BES investors will be exposed to the risk of insufficient revenue to recover their investment since the estimated BES lifetimes for worst case scenario (11 and 9 years respectively) are lower than the payback period of 12 years. In addition, the remaining two business models will hardly be profitable to storage investors, even assuming the best case scenario, as their estimated lifetimes are shorter than their respective payback period. In summary, this study can assist storage investors in evaluating the potential economic outcomes of BES investment across different business models, particularly for business models offering balancing services such as frequency response.

\section{F. Revenue Required for Positive NPV}

To fully understand the economics of BES investments and devise appropriate business models, this section presents a summarized analysis on the revenue required to achieve a positive return on investment with three different payback periods. In this context, Fig. 8 shows for 5, 10 and 15 years' payback period and different technology costs, the revenue that an investor would require to achieve a positive NPV.

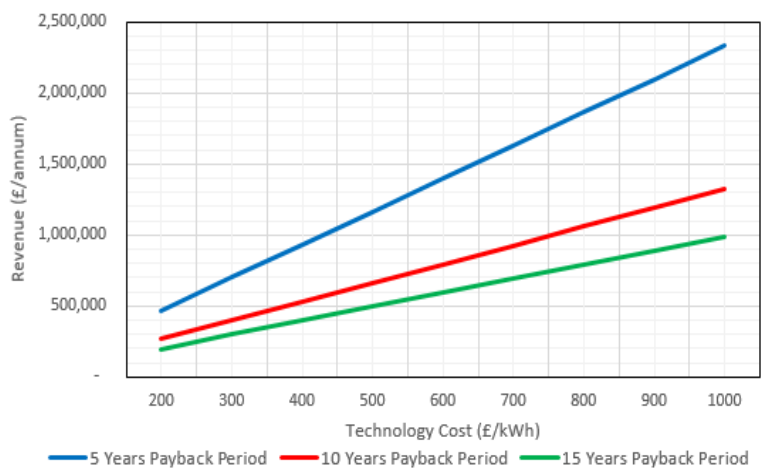

Fig. 8. Minimum annual revenue for achieving a positive NPV. 
The results show that a minimum annual revenue of approximately $700,000 \mathrm{f}$ /annum is required by storage investors to recover the investment of a $300 £ / \mathrm{kWh}$ BES technology within 5 years. If a 10 year payback period is assumed, then only $400,000 \mathrm{f}$ /annum is necessary to achieve return of investment.

\section{CONCLUSION}

This paper has presented a systematic way to analyze the economic viability of BES investments, particularly when considering multi-service business models. In summary, a BES investment model has been developed to perform an economic analysis of various BES business models under current market arrangements.

The results have indicated that a multi-service business model can offer the best financial benefit for storage investors, i.e. the highest NPV as well as the shortest payback period. However, we have also demonstrated that provision of network services or seizing arbitrage opportunities in the energy market yield a low revenue and are likely to undermine the business case of BES. These findings are significant to storage investors, which will enable them to thoroughly analyze the potential risks before considering a BES investment and which business model to pursue to ensure positive returns.

In addition, provision of frequency response service is the highest remunerating single service (even when compared to a combined provision of network services and energy arbitrage). Although, when analyzing the potential degradation effects due to frequent charge/discharge cycles, provision of frequency response only can seriously undermine the business case and result in a loss of capital invested.

Overall, the proposed model can promote deployment of BES technologies by enhancing the understanding of the economic viability of BES investments, taking into consideration various business models and characteristics of BES, such as technology cost and lifetime. For further studies, it would be advantageous to conduct an economic analysis of BES across different European markets and even consider the potential of investing in hybrid technologies, for example high power delivery technologies (e.g. supercapacitors) combined with high energy technologies (e.g. compressed air energy storage).

\section{ACKNOWLEDGMENT}

The authors gratefully acknowledge the contributions from UK Power Networks in providing relevant data for this study.

\section{REFERENCES}

[1] "Energy Storage in the UK: An Overview," Renewable Energy Association, 2015.

[2] D. Pudjianto, M. Aunedi, P. Djapic and G. Strbac, "Whole-Systems Assessment of the Value of Energy Storage in Low-Carbon Electricity Systems," IEEE Transactions On Smart Grid, vol. 5, no. 2, pp. 1098$1109,2014$.
[3] "Development of Electricity Storage in the National Interest," The Electricity Storage Network, 2014.

[4] A. Goater, "Energy Storage," The Parliamentary Office of Science and Technology, 2015.

[5] T. Sercan, "Energy Storage Overview : Applications, Technologies and Economical Evaluation," Quanta Technology, 2011.

[6] D. Akinyele and R. Rayudu, "Review of energy storage technologies for sustainable networks," Sustainable Energy Technologies and Assessments, no. 8, pp. 74-91, 2014.

[7] N. Wade, P. Taylor, P. Lang and P. Jones, "Evaluating the benefits of an electrical energy storage system in a future smart grid," Energy Policy, no. 38, pp. 71807188, 2010.

[8] R. Moreira, R. Moreno and G. Strbac, "Synergies and conflicts among energy storage services," in IEE International Energy Conference Energycon, Leuven, 2016.

[9] S. S. T. Maciej.S, "Primary Frequency Regulation with Li-ion Battery Storage System: a Case Study for Denmark," pp. 487-492, 2013.

[10] R. Lopez and J. Augustin, "Techno-economic analysis of grid-connected battery storage," Energy Conversion and Management, no. 91, pp. 394-404, 2015.

[11] A. Oudalov, D. Chartouni, C. Ohler and G. Linhofer, "Value Analysis of Battery Energy Storage Applications in Power Systems," pp. 2206-2211, 2006.

[12] R. Moreno, R. Moreira and G. Strbac, "A MILP model for optimising multi-service portfolios of distributed energy storage," Applied Energy, no. 137, pp. 554566, 2015.

[13] V. Stoiljkovic, "Comparing engineering projects by financial return," IEEE Potentials, pp. 17-21, 2010.

[14] J. Pasqual, E. Padilla and E. Jadotte, "Technical note: Equivalence of different profitability criteria with the net present value," Int. J. Production Economics, no. 142, pp. 205-210, 2013.

[15] R. Kempener and E. Borden, "Battery Storage For Renewables: Market Status and Technology Outlook," IRENA, 2015.

[16] "Saft," May 2014. [Online]. Available: www.saftbatteries.com.

[17] L. Jianwei, M. Anthony, Z. Min and Y. Weijia, "Analysis of battery lifetime extension in a SMESbattery hybrid energy storage system using a novel battery lifetime model," Energy, no. 86, pp. 175-185, 2015. 